\title{
Organic-Inorganic Hybrid Gate Dielectrics Using Self-Assembled Multilayers For Low-Voltage Operating Thin-Film Transistors
}

\author{
Yong-Wan Kim and Young-Geun $\mathrm{Ha}^{*}$ \\ Department of Chemistry, Kyonggi University, Gyeonggi-Do 443-760, Republic of Korea
}

\begin{abstract}
Advanced electronic materials have attracted great interest for their potential use in flexible, largearea, and printable electronic applications. However, fabricating high-performance low-voltage thin-film transistors (TFTs) for those applications with these advanced semiconductors is still challenging because of a lack of dielectric materials which satisfy both the required electrical and physical performance. In this work, we report self-assembled hybrid multilayer gate dielectrics prepared using a facile solution procedure to achieve organic semiconductor and amorphous oxide semiconductor-based thin-film transistors with ultralow operating voltage. These self-assembled hybrid multilayer gate dielectrics were constructed by iterative selfassembly of synthesized bifunctional phosphonic acid-based organic molecules and ultrathin high-k hafnium oxide layers. The novel self-assembled hybrid multilayer gate dielectrics exhibit excellent dielectric properties with exceptionally large capacitances (up to $815 \mathrm{nF} / \mathrm{cm}^{2}$ ) and low-level leakage current densities of $<1.56$ $\times 10^{-6} \mathrm{~A} / \mathrm{cm}^{2}$, featureless morphology (RMS roughness $<0.24 \mathrm{~nm}$ ), and thermal stability (up to $300{ }^{\circ} \mathrm{C}$ ). Consequently, these hybrid gate dielectrics can be incorporated into thin-film transistors with pentacene as p-type organic semiconductors, and with indium oxide as n-type inorganic semiconductors. The resulting TFTs functioned at ultralow voltages $\left(< \pm 2 \mathrm{~V}\right.$ ) and achieved high transistor performances (hole mobility: $0.88 \mathrm{~cm}^{2} /$ $\mathrm{V}: \mathrm{s}$, electron mobility: $7.8 \mathrm{~cm}^{2} / \mathrm{V} \cdot \mathrm{s}$ and on/off current ratio $>10^{4}$, and threshold voltage: $\pm 0.5 \mathrm{~V}$ ).
\end{abstract}

(Received 4 November 2021; Accepted 18 November 2021)

Keywords: self-assembled multilayer, hybrid gate dielectric, TFTs, ultralow-voltage operation

\section{Introduction}

Wearable biosensors built from innovative materials have attracted significant attention in recent years because of their prospective applications, including flexible electronics, thinfilm transistors (TFTs), or radio-frequency identification (RFID) [1-7]. Among advanced semiconductors, organic and polymer materials have important advantages, such as low cost and flexibility. To achieve a level of high performance the TFTs must have adequate source-drain current $\left(\mathrm{I}_{\mathrm{SD}}\right)$ and operate in the ultralow-voltage range $(< \pm 2 \mathrm{~V})$. Despite a significant number of improvements, the large operating voltages of organic and polymeric semiconductor-based TFTs, with intrinsically low semiconductor mobilities, impede their further application [8]. To achieve TFTs with

- 하영근: 교수, 김용완: 석사과정

*Corresponding Author:

[Tel: +82-31-249-9635, E-mail: ygha@kgu.ac.kr]

Copyright (c) The Korean Institute of Metals and Materials the optimum low voltage operation, the capacitance per unit area of gate dielectric $\left(C_{i}\right)$ should be increased, either by enhancing the dielectric constant $(k)$ or by reducing the thickness (d) of the dielectric film. Capacitance is determined using Eq. 1, where $d$ is the film thickness, $k$ is the dielectric constant of the material, and $\varepsilon_{0}$ is the vacuum permittivity.

$$
C_{i}=\varepsilon_{0} \frac{k}{d}
$$

A typical approach to increase the capacitance of dielectrics is to utilize high-dielectric-constant materials, such as zirconia, hafnia, and alumina [9]. However, the hightemperature process (over $400{ }^{\circ} \mathrm{C}$ ) required to achieve electric properties suitable for gate dielectrics has been a major challenge in the development of flexible electronic devices on plastic substrates [10]. Alternatively, by reducing the thickness of the dielectric film, nanoscopic selfassembled mono- or multi-layer dielectrics can be produced which exhibit enhanced capacitance. In addition, such 
dielectrics can be fabricated using solution-phase processing at low temperatures, thereby reducing fabrication costs [11-12].

This research group previously reported novel selfassembled multilayer hybrid dielectrics fabricated by the iterative stacking of self-assembled organic layers between high- $k$ oxide thin films [13]. With their lower thickness and higher dielectric constant these dielectric films exhibited dramatically enhanced capacitance. However, a complicated multi-step fabrication process was required to ensure adequate insulation, as the self-assembled organic layers have a low film density because of the short length of the hydrocarbons. Therefore, to achieve excellent insulation with large capacitance while reducing the cost of multi-step processing, the density of the self-assembled organic layers must be improved.

In this study, we synthesized hydrocarbon-based bifunctional phosphonic acid with longer chains (C10; ten carbon atoms) than those used in previous studies (C8; eight carbon atoms). We fabricated organic-inorganic hybrid multilayers with efficient and dense self-assembled organic layers between high- $k$ hafnium oxide thin layers to improve the insulating properties of the gate dielectrics. Consequently, the fabricated hybrid dielectrics exhibited excellent dielectric properties, high thermal stability, and a smooth surface despite the one-layer deposition of bifunctional organic molecules in the hybrid layer. Moreover, the surface characteristics of these hybrid films can be modified by facile solution dipping of the hydrocarbon self-assembled monolayers (SAMs). Subsequently, the hybrid dielectric films were incorporated with various types of organic and inorganic semiconductors, to demonstrate the dielectric in an ultralow voltage operating TFT.

\section{Experimental Details}

Materials. The precursor solutions for hafnium oxide were prepared using commercially available hafnium (IV) chloride (Aldrich). n-octadecyl phosphonic acid (ODPA) from Alfa Aesar was used to prepare the hydrophobic SAMs. Pentacene (TCI) was employed as an organic semiconductor without additional purification. Indium (III) nitrate hydrate (99.9\%, Aldrich) was used as the precursor to form indium oxide thin films.

\section{Fabrication and characterization of the self-assembled} hybrid gate dielectric films. $\mathrm{n}^{++}$-silicon wafers, as the gate electrode and substrate, were treated by ultrasonic cleaning in EtOH for 2 min and blown with an $\mathrm{N}_{2}$ stream to dry. Then, the substrates were UV-ozone cleaned for $5 \mathrm{~min}$ to improve their wettability. A precursor solution of $0.1 \mathrm{M}$ hafnium (IV) chloride $\left(\mathrm{HfCl}_{4}\right)$ in ethanol was prepared, and nitric acid $\left(70 \%\right.$, molar ratios of $\mathrm{HfCl}_{4}$ and $\left.\mathrm{HNO}_{3}=1: 10\right)$ were mixed. The precursor was stirred for hafnium sol hydrolysis at $50{ }^{\circ} \mathrm{C}$ for $4 \mathrm{~h}$. To fabricate the first $\mathrm{HfO}_{\mathrm{x}}$ base layer, the $0.01 \mathrm{M}$ $\mathrm{HfCl}_{4}$ solution was applied (5000 rpm, $40 \mathrm{~s}$ ) to a Si substrate, and annealed at $150{ }^{\circ} \mathrm{C}$ for 20 min to produce the $\mathrm{HfO}_{\mathrm{x}}$ base thin film. Afterward, 1,12-dodecanediylbis (phosphonic acid) (DDBPA), a bifunctional phosphonic acid-based organic molecule, was prepared using a two-step procedure and was synthesized and characterized by conventional analytical/ spectroscopic techniques (see the Supporting Information for the synthetic and characterization details).

For the DDBPA deposition in the hybrid multilayer, the base $\mathrm{HfO}_{\mathrm{x}}$ thin-film coated substrate was immersed into 1.0 $\mathrm{mM}$ of DDBPA in ethanol for $30 \mathrm{~min}$. After cleaning in ethanol, the DDBPA-coated substrate was blown with an $\mathrm{N}_{2}$ stream to dry. To complete one layer of the hybrid multilayer ( $1 \mathrm{~L})$, another hafnium precursor solution was applied, and the coated substrate was cured at $150{ }^{\circ} \mathrm{C}$ for $20 \mathrm{~min}$ to produce a $\mathrm{HfO}_{\mathrm{x}}$ top thin layer. To form two layers of the hybrid multilayer $(2 \mathrm{~L})$, the successive self-assembly of DDBPA and spin-coating of $\mathrm{HfO}_{\mathrm{x}}$ was repeated. For compatibility with an organic semiconductor, $1 \mathrm{~L}$ was finally dipped in $2.0 \mathrm{mM}$ of n-octadecyl phosphonic acid (ODPA) in ethanol for $1 \mathrm{~h}$. The dipped substrates were cleaned with copious amounts of ethanol and then blown under an $\mathrm{N}_{2}$ stream. The surface morphologies of the fabricated gate dielectric films were assessed using atomic force microscopy (AFM, PSIA XE-150). The surface properties of the dielectrics were analyzed using a commercial static water contact angle (WCA) system (Phoenix-I, SEO).

Fabrication and characterization of capacitor and transistor devices using the self-assembled hybrid multilayer gate dielectrics. To assess the electrical characteristics of the gate dielectrics, MIS structures were constructed using aluminum ( $\mathrm{Al}$ ) dots placed directly onto the self-assembled hybrid dielectrics using a shadow mask 
under ultrahigh vacuum (UHV; $\sim 10^{-6}$ Torr). To characterize the performance of the TFTs containing the self-assembled hybrid dielectrics, organic TFTs were constructed with a 40 $\mathrm{nm}$ pentacene thin film on the hybrid dielectrics, followed by gold $(40 \mathrm{~nm})$ as source/drain electrodes using a shadow mask under UHV. For the precursor of the amorphous oxide semiconductor, $0.1 \mathrm{M}$ of indium nitrate hydrate solution in deionized water was prepared and was deposited onto the self-assembled hybrid multilayer dielectrics via a spincoating method. The deposited film was then thermally treated at $250{ }^{\circ} \mathrm{C}$ for $30 \mathrm{~min}$. To achieve the transistor structure, source/drain $\mathrm{Al}$ electrodes were deposited through a shadow mask under UHV.

The current-voltage (I-V) properties of the TFTs were assessed using an HP4145B semiconductor parameter analyzer. Capacitance was assessed using an HP 4192A impedance analyzer.

\section{Results and Discussion}

We characterized the solution-phase processed hybrid dielectric films with bifunctional self-assembled organic molecules and ultrathin high- $k$ hafnium oxide. To confirm their surface energy and uniformity, the hybrid dielectric films were analyzed using standard surface characterization methods. In this way we demonstrated that the hybrid film formation procedure resulted in thermally stable, surfaceenergy-tunable, and smooth films, resulting in excellent dielectric properties including high capacitance and insulating performance. Finally, the prepared hybrid multilayer gate dielectrics were integrated with an organic semiconductor (pentacene) and an amorphous oxide semiconductor (indium oxide) to demonstrate ultralowvoltage TFTs.

\subsection{Characteristics of the self-assembled hybrid multilayer dielectrics}

In general, hydrocarbon SAMs that include fewer than ten carbons produce disorganized molecular layers, due to insufficient van der Waals molecular interactions among the SAMs [14]. To increase the density of the self-assembled organic layers in hybrid films to improve their dielectric properties, our previous bifunctional phosphonic acid-based (a)

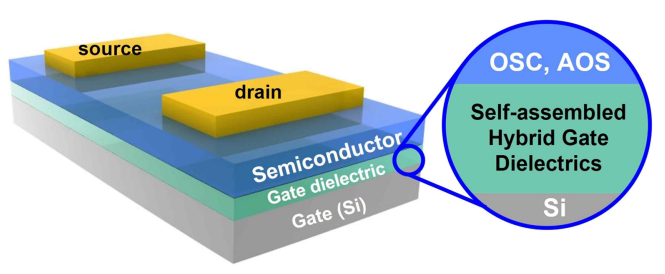

(b)

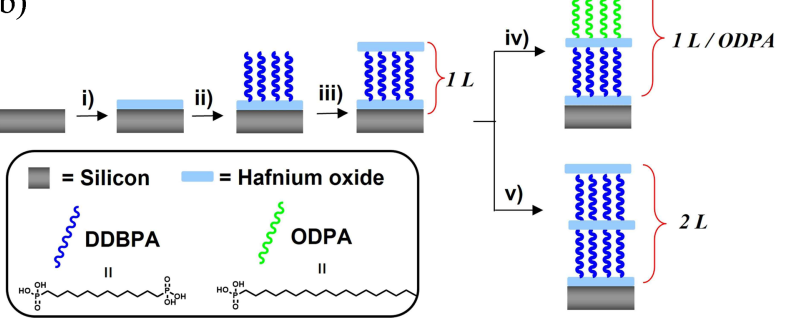

Fig. 1. (a) Schematic view of TFT device structure using selfassembled multilayer hybrid dielectrics. (b) Fabrication procedure of self-assembled hybrid dielectrics (i) applying $\mathrm{HfO}_{\mathrm{x}}$ first base layer, (ii) formation of self-assembly of DDBPA, (iii) applying $\mathrm{HfO}_{\mathrm{x}}$ top layer, (iv) self-assembly of ODPA for $1 \mathrm{~L} / \mathrm{ODPA}$, and (v) DDBPA and another $\mathrm{HfO}_{\mathrm{x}}$ layer to complete $2 \mathrm{~L}$.

molecules (including eight carbon atoms) [13] were modified with DDBPA with longer hydrocarbon chains (ten carbon atoms), which generally show ordered and dense molecular assemblies.

To generate the hybrid multilayer dielectric, DDBPA with bifunctional phosphonic acid was self-assembled between the phosphonate and $\mathrm{HfO}_{\mathrm{x}}$ layers by coordination of the phosphonate and hafnium oxides. On the first $\mathrm{HfO}_{\mathrm{x}}$ base layer on the Si substrate, a hybrid bilayer of DDBPA/HfOx was successively stacked to produce hybrid multilayers with different thicknesses (1 L: DDBPA/ $\mathrm{HfO}_{\mathrm{x}}$, and $2 \mathrm{~L}$ : DDBPA/ $\mathrm{HfO}_{\mathrm{x}} / \mathrm{DDBPA} / \mathrm{HfO}_{\mathrm{x}}$ ) (Fig. 1).

For organic semiconductor-based TFTs, one layer of the hybrid multilayer $(1 \mathrm{~L})$ was chosen as the gate dielectric. The hydrocarbon-based phosphonic acid (n-octadecyl phosphonic acid; ODPA) self-assembled owing to the hydrophilicity of the top $\mathrm{HfO}_{\mathrm{x}}$ layer, due to both reduced surface energy and increased insulating property. The top surface of the hybrid multilayer was $\mathrm{HfO}_{\mathrm{x}}$, which is a hydrophilic surface with high surface energy due to hydroxyl groups (-OH) on the metal oxide surface. The - $\mathrm{OH}$ groups negatively affect the operation of the TFTs because the hydroxyl functional groups are positive charge traps [15].

To overcome this limitation, hydrocarbon-based SAMs were deposited on the surface of the dielectrics to effectively 
(a)

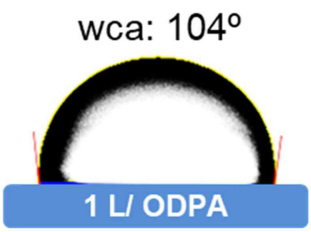

wca: $23^{\circ}$

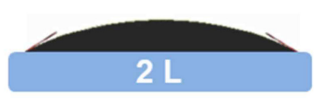

(b)

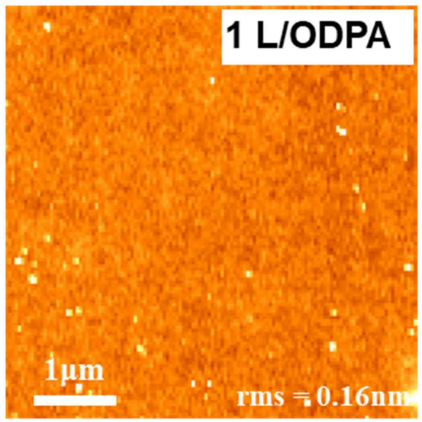

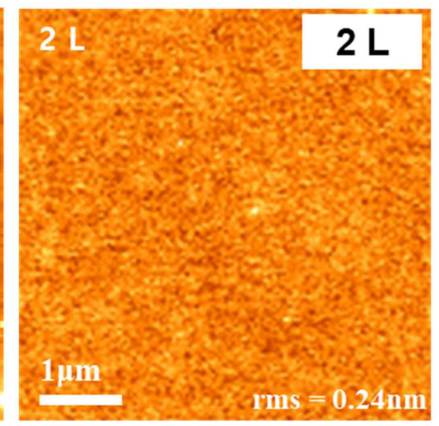

(c)

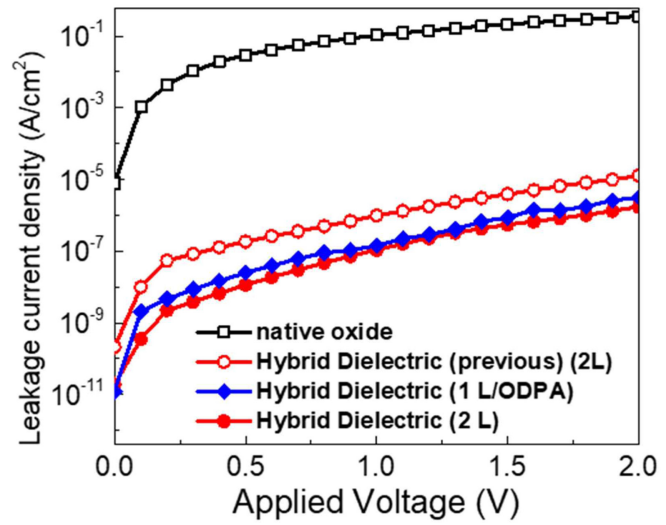

(d)

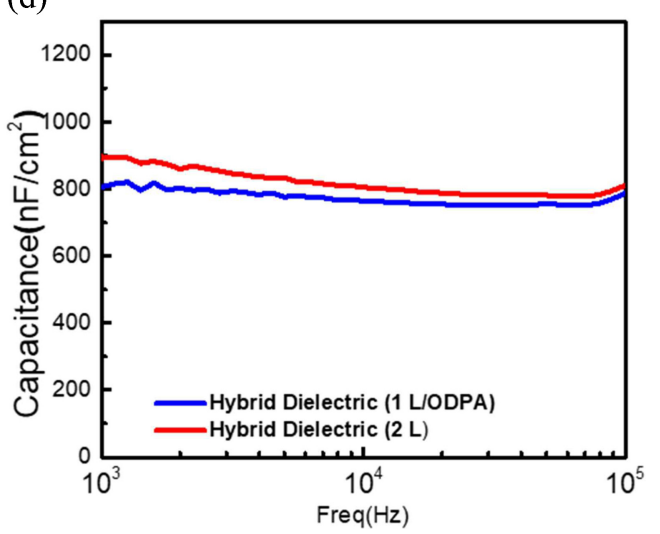

Fig. 2. (a) WCAs and (b) AFM images with different self-assembled hybrid multilayer surfaces (1 L/ODPA and 2 L). (c) Leakage current densities vs. applied voltage and (d) capacitance-frequency properties of self-assembled multilayer hybrid dielectrics.

remove the hydroxyl groups. Moreover, the low surface energy characteristic of the hybrid dielectric films improved compatibility between the dielectric surface and organic semiconductors during deposition. This influences the arrangement of the organic semiconductor molecules, and thus the performance of the TFTs [16]. In addition to lowering the surface energy, the additional layer of hydrophobic SAMs on the dielectrics enhances the insulating properties of dielectric films by providing an additional leakage barrier, resulting in improved performance of organic thin-film transistors (OTFT) devices [17].

For these reasons, an ODPA with long hydrocarbon chains, providing the synergistic benefits of a well-ordered organic semiconductor with insulating properties, was utilized to improve the operating efficiency of the organic TFTs. After the application of the ODPA layers on $1 \mathrm{~L}$ (1 L/ODPA), the surface property was dramatically changed to hydrophobic $\left(104^{\circ}\right)$, compared to the bare oxide surface (Fig. 2a). For the solution-processable amorphous oxide semiconductor-based
TFT, $2 \mathrm{~L}$ was used as the gate dielectric to maintain the acceptable insulating property of the hybrid film after thermal annealing during oxide semiconductor film formation. The surface smoothness, which is an important factor affecting the performance of TFTs, was analyzed using AFM. Fig. 2b shows AFM images of the self-assembled hybrid dielectrics (1 L/ODPA and $2 \mathrm{~L}$ ), which are featureless with pinhole-free surfaces (RMS roughness $<0.24 \mathrm{~nm}$ ), indicating that the solution-processed hybrid multilayer films had very smooth surfaces.

To evaluate the dielectric performances of the selected hybrid dielectric films (1 L/ODPA for OTFTs and $2 \mathrm{~L}$ for AOS-TFTs) in organic and amorphous oxide semiconductor TFTs, the insulating property and capacitance of the MIS capacitors were then assessed for each of the hybrid dielectrics (Figs. 2c and 2d). Fig. 2c shows the leakage current and applied voltage data for the hybrid dielectrics. Compared with the native oxide layer, which had extremely low dielectric strength, the leakage currents of the self- 
Table 1. Device properties of hybrid dielectric films and TFTs with hybrid dielectrics.

\begin{tabular}{|c|c|c|c|c|c|c|c|}
\hline \multirow{2}{*}{$\begin{array}{l}\text { Hybrid Multilayer } \\
\text { Gate Dielectrics }\end{array}$} & \multicolumn{3}{|c|}{ Dielectric property } & \multicolumn{4}{|c|}{ TFT } \\
\hline & $\begin{array}{c}\text { Capacitance } \\
\left(\mathrm{nF} / \mathrm{cm}^{2}\right)\end{array}$ & $\begin{array}{l}\text { Leakage current } \\
\left(\mathrm{A} / \mathrm{cm}^{2}\right)\end{array}$ & $\operatorname{WCA}\left({ }^{\circ}\right)$ & Semiconductor & $\begin{array}{l}\text { Mobility } \\
\left(\mathrm{cm}^{2} \mathrm{~V}^{-1} \mathrm{~s}^{-1}\right)\end{array}$ & $\begin{array}{l}\text { On/off } \\
\text { ratio }\end{array}$ & $\mathrm{V}_{\mathrm{th}}(\mathrm{V})$ \\
\hline $1 \mathrm{~L} / \mathrm{ODPA}$ & 800 & $3.1610^{-6}$ & 104 & pentacene & 0.88 & $10^{5}$ & -0.6 \\
\hline $2 \mathrm{~L}$ & 815 & $1.5610^{-6}$ & 23 & $\mathrm{In}_{2} \mathrm{O}_{3}$ & 7.8 & $10^{4}$ & 0.5 \\
\hline
\end{tabular}

assembled dielectric films were dramatically lower in the same voltage range because of the excellent insulating properties of the hybrid layers.

Furthermore, the leakage current densities of DDBPAbased hybrid multilayer dielectrics (1 L/ODPA and $2 \mathrm{~L}$ ) were 10 times lower than those in a previous study using shorter organic (C8)-based hybrid multilayers at the same operating voltage $(2 \mathrm{~V})$, confirming that denser organic layers were assembled in each bilayer of the hybrid. Consequently, the longer organic molecules improved the insulating property (C10) thanks to the leakage barrier of the denser organic films. Note that all of the self-assembled hybrid gate dielectrics have low leakage currents which are suitable for TFT operations. The hybrid film (1 L/ODPA) for the OTFTs exhibited a relatively smaller capacitance $\left(800 \mathrm{nF} / \mathrm{cm}^{2}\right)$ than the hybrid dielectrics (2 L) for AOS-TFTs $\left(815 \mathrm{nF} / \mathrm{cm}^{2}\right)$ (Fig. $2 \mathrm{~d})$. This is because the organic ODPA has an intrinsically low dielectric property compared to that of the $\mathrm{HfO}_{\mathrm{x}}$ inorganic layer.[18] However, the capacitance of these films was large enough because of the nanoscopic thin layer, resulting in ultralow-voltage TFTs.

The dielectric performance data of the hybrid gate dielectrics are summarized in Table 1. Because the selfassembled hybrid dielectrics with dense organic molecule assemblies showed excellent properties, including excellent insulating performance, capacitance, and smooth surfaces, they were employed as gate dielectrics in TFTs with ultralow operating voltages.

Amorphous oxide semiconductor films require an annealing procedure. To confirm their formation, the thermal stability of the hybrid gate dielectric films was examined in the MIS capacitor structure, by measuring the leakage current density as a function of applied voltage after various thermal treatments (up to $300{ }^{\circ} \mathrm{C}$ ). Fig. 3 plots the $J$ - $V$, indicating the hybrid films exhibited comparable insulating performance under a similar voltage range $(-2 \mathrm{~V}$ to $+2 \mathrm{~V})$ before and after

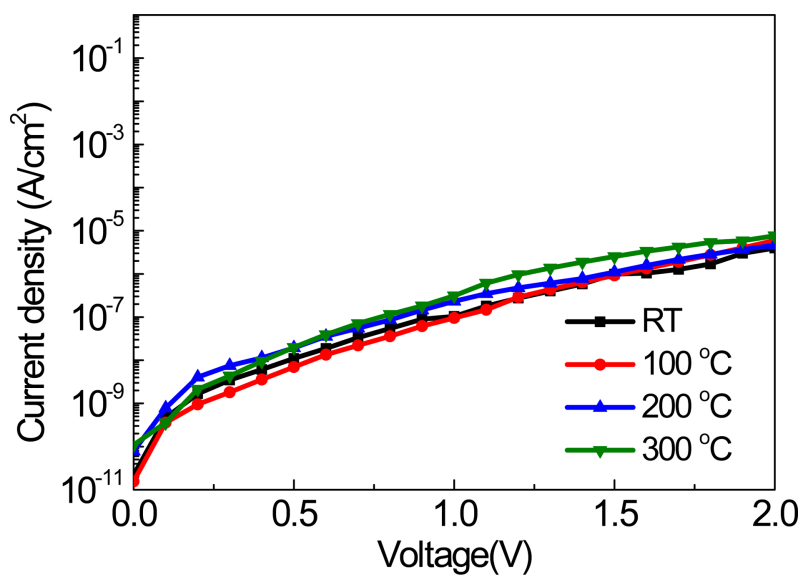

Fig. 3. Leakage current density vs. voltage plots for post-annealing of self-assembled hybrid gate dielectrics.

being heat-treated up to $300{ }^{\circ} \mathrm{C}$. These results clearly show that the hybrid films properties have both thermal and dielectric stability, making them suitable for hightemperature processes such as inorganic semiconductor integration.

\subsection{Characteristics of TFTs fabricated using} the self-assembled hybrid multilayer dielectrics

We next fabricated TFTs with pentacene and indium oxide as representative organic and amorphous oxide semiconductors to verify the compatibility of our selfassembled hybrid dielectrics with different semiconductor materials. Table 1 shows the performances of the organic and amorphous oxide TFTs employing the self-assembled hybrid dielectrics. Based on the measured electrical performances, the OTFTs with self-assembled hybrid dielectrics (1 L/ ODPA) showed excellent performance, including great mobility of $0.88 \mathrm{~cm}^{2} / \mathrm{V} \cdot \mathrm{s}$ and an $\mathrm{I}_{\text {on }} / \mathrm{I}_{\text {off }}$ of $10^{5}$ at ultralow voltage operation with a low $\mathrm{V}_{\text {th }}$ of $-0.6 \mathrm{~V}$.

For the amorphous oxide semiconductor-based TFTs, the indium oxide TFTs with self-assembled multilayer dielectrics (2 L) showed the best device performance. They exhibited 

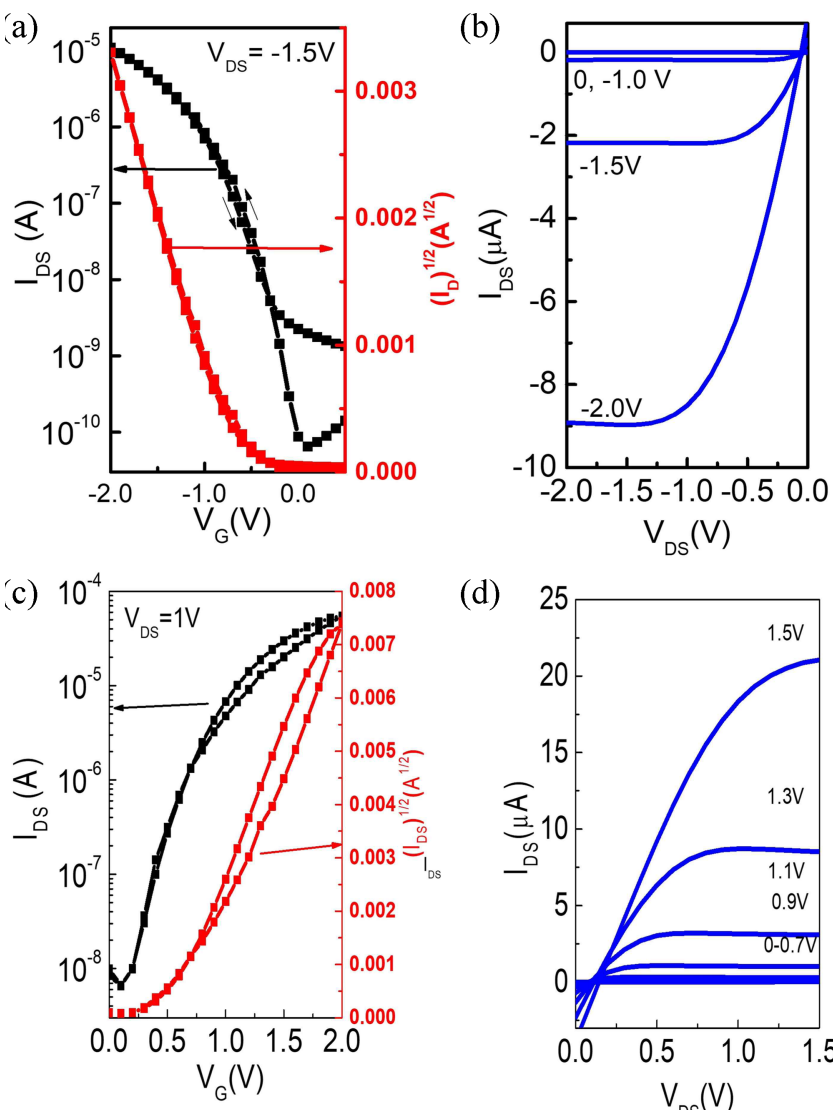

(d)

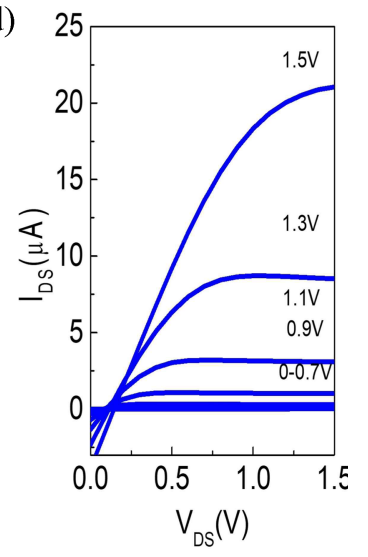

Fig. 4. Device performances of TFTs with self-assembled hybrid dielectrics. (a) Output and (b) transfer plots of organic TFTs with hybrid multilayer gate dielectrics (1 L/ODPA). (c) Output and (d) transfer plots of amorphous oxide TFTs with hybrid multilayer gate dielectrics $(2 \mathrm{~L})$. $\left(\mathrm{I}_{\mathrm{DS}}\right.$ :drain-source current, $\mathrm{V}_{\mathrm{G}}$ : gate voltage, $\mathrm{V}_{\mathrm{DS}}$ : drain-source voltage)

excellent $I-V$ characteristics: mobility $\left(\mu_{\mathrm{e}}\right)$ of $7.84 \mathrm{~cm}^{2} / \mathrm{V} \cdot \mathrm{s}$, $\mathrm{I}_{\text {on }} / \mathrm{I}_{\text {off }}$ of $\sim 10^{4}$, and $\mathrm{V}_{\text {th }}$ of $0.5 \mathrm{~V}$, better performance than the TFTs with $\mathrm{HfO}_{\mathrm{x}}$ dielectrics thermally annealed at $400{ }^{\circ} \mathrm{C}$ (mobility of $\sim 1.65 \mathrm{~cm}^{2} / \mathrm{V} \cdot \mathrm{s}, \mathrm{I}_{\text {on }} / \mathrm{I}_{\text {off }}$ of $\sim 10^{5}$, and $\mathrm{V}_{\text {th }}$ of $0.3 \mathrm{~V}$ ).

Fig. 4 shows the standard electrical behaviors of the OTFTs operating at ultralow voltages. These characteristics exhibited trivial hysteresis and were reproducible after 20 repeated operations. The average mobility was determined using the transfer data plot (Figs. $4 \mathrm{a}$ and $4 \mathrm{c}$ ) using the square root of $I_{D}$ vs. $V_{S D}[19]$.

\section{Conclusion}

We demonstrated a facile solution process to fabricate novel self-assembled hybrid multilayer dielectrics using phosphonic acid-based molecules and ultrathin hafnium oxides. The self-assembled hybrid multilayer dielectric films had smooth, pinhole-free surfaces and exhibited excellent thermal stability, capacitance, and insulating properties. The surface chemical properties of the dielectric films can be modified using hydrophobic phosphonic acid. Consequently, tuning the surface properties of the dielectric films made them compatible with various semiconductors, such as organic and amorphous oxide. A well-organized thin film was constructed on the hybrid dielectrics, resulting in excellent TFT performances at ultralow operating voltages.

\section{Acknowledgments}

This work was supported by the Kyonggi University Graduate Research Assistantship 2020, and the Basic Science Research Program (2020R1F1A1062765) of the National Research Foundation of Korea (NRF).

\section{REFERENCES}

1. H. Klauk, Chem. Soc. Rev. 39, 2643 (2010).

2. H. Sirringhaus, $A d v$. Mater. 26, 1319 (2014).

3. Y. Zang, F. Zhang, D. Huang, X. Gao, C. Di, and D. Zhu, Nat. Commun. 6, 6269 (2015).

4. X. Yu, T. J. Marks, and A. Facchetti, Nat. Mater. 15, 383 (2016).

5. A. Miyamoto, S. Lee, N. F. Cooray, S. Lee, M. Mori, N. Matsuhisa, H. Jin, L. Yoda, T. Yokota, A. Itoh, M. Sekino, H. Kawasaki, T. Ebihara, M. Amagai, and T. Someya, Nat. Nanotechnol. 12, 907 (2017).

6. W.-S. Choi, Korean J. Met. Mater. 59, 314 (2021).

7. S.-H. Choe, J.-K. Jang, H.-J. Kim, J.-W. Choi, S.-B. Heo, Y.-S. Kim, Y.-M. Kong, and D. Kim, Korean J. Met. Mater. 59, 545 (2021).

8. Y. G. Ha, K. Everaerts, M. C. Hersam, and T. J. Marks, Acc. Chem. Res. 47, 1019 (2014).

9. R. P. Ortiz, A. Facchetti, and T. J. Marks, Chem. Rev. 110, 205 (2010).

10. J. Robertson, Reports Prog. Phys. 69, 327 (2006).

11. H. Ma, O. Acton, D. O. Hutchins, N. Cernetic, and A. K.-Y. Jen, Phys. Chem. Chem. Phys. 14, 14110 (2012).

12. M. J. Kim, K. Pak, W. S. Hwang, S. G. Im, and B. J. Cho, ACS Appl. Mater. Interfaces 10, 37326 (2018).

13. H. R. Byun, E. A. You, and Y. G. Ha, ACS Appl. Mater. 
Interfaces 9, 7347 (2017).

14. J. B. Brzoska, N. Shahidzadeh, and F. Rondelez, Nature 360, 719 (1992).

15. M.-H. Yoon, C. Kim, A. Facchetti, and T. J. Marks, J. Am Chem. Soc. 128, 12851 (2006).

16. I. N. Hulea, S. Fratini, H. Xie, C. L. Mulder, N. N. Iossad, G. Rastelli, S. Ciuchi, and A. F. Morpurgo, Nat. Mater. 5,
$982(2006)$

17. H. Klauk, U. Zschieschang, J. Pflaum, and M. Halik, Nature 445, 745 (2007).

18. M. Xiao, C. Qiu, Z. Zhang, and L. M. Peng, ACS Appl. Mater. Interfaces 9, 34050 (2017).

19. G. Horowitz and M. E. Hajlaoui, Adv. Mater. 12, 1046 (2000). 